\title{
Robotic resection of a mediastinal hydatic cyst and diaphragmatic repair: a case report
}

\author{
Sotirios Georgios Popeskou ${ }^{1}$, Jonathan Douissard ${ }^{1}$, Niki Christou ${ }^{1}$, Giacomo Puppa ${ }^{2}$, Frederic Ris ${ }^{1}$, \\ Nicolas Christian Buchs ${ }^{1}$, Minoa Karin Jung ${ }^{1}$, Christian Toso ${ }^{1}$ \\ ${ }^{1}$ Department of Visceral Surgery and Transplantation, ${ }^{2}$ Department of Pathology, Geneva University Hospitals, Geneva, Switzerland \\ Correspondence to: Sotirios Georgios Popeskou. Department of Visceral Surgery and Transplantation, Geneva University Hospitals, Geneva, \\ Switzerland. Email: salvator10@yahoo.com.
}

\begin{abstract}
This is the case of a 52-year-old man with an unusual presentation of a hydatid cyst. The patient was diagnosed with a diaphragmatic paralysis over 20 years ago and presented an ever-increasing difficulty in breathing. A mediastinal mass traversing the left diaphragm was identified and a subsequent robotic resection with synchronous diaphragmatic repair was performed. To our knowledge this is the first case of a hydatid cyst resection with concomitant diaphragmatic repair using a robotic platform, without which we believe an open laparotomy would have been the operation of choice, given the technical difficulties of a standard laparoscopy. The patient has had an uneventful recovery, discharged on the fifth day post-op and his respiratory symptoms have completely regressed.
\end{abstract}

Keywords: Robotic surgery; hydatic cyst; diaphragmatic repair; case report

Received: 24 November 2019; Accepted: 19 February 2020; Published: 05 October 2020.

doi: 10.21037/jovs.2020.03.01

View this article at: http://dx.doi.org/10.21037/jovs.2020.03.01

\section{Introduction}

Cystic echinococcosis is a widely endemic helminthic disease caused by infection with metacestodes of the Echinococcus granulosus tapeworm (1). Upon infection, cyst formation mainly occurs in the liver $(70 \%)$ or the lungs $(20 \%)$ and diagnosis involves serum serologic testing for antibodies against hydatid antigens, but preferably imaging by ultrasound or CT/MRI (1). E. granulosus infections usually present as solitary cysts, and mainly have singleorgan involvement (2). Our case is unique as far as its rare presentation and minimally invasive therapeutic approach using a robotic platform is concerned.

\section{Case presentation}

All procedures performed in this study were in accordance with the ethical standards of the institutional and/or national research committee(s) and with the Helsinki Declaration (as revised in 2013). Oral informed consent has been acquired as the patient was not physically available

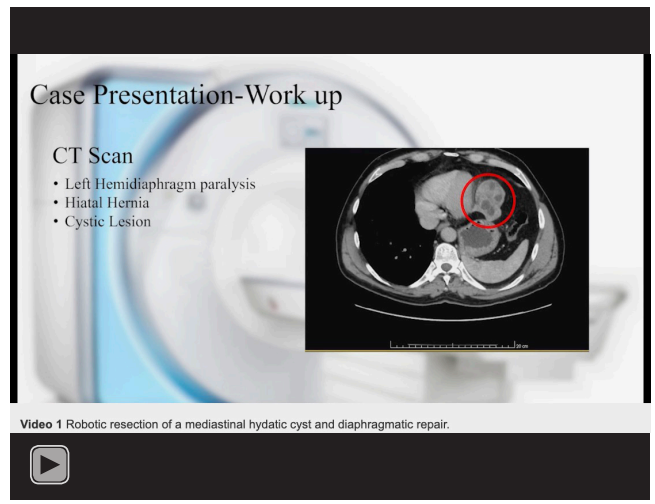

Video 1 Robotic resection of a mediastinal hydatic cyst and diaphragmatic repair.

during the creation of this paper.

In this video we present the case of a 52-year-old man of East European descent, diagnosed with left diaphragmatic paralysis over 20 years ago (Video 1 ). The patient is otherwise in excellent health, without any previous operations and under no regular medication. 


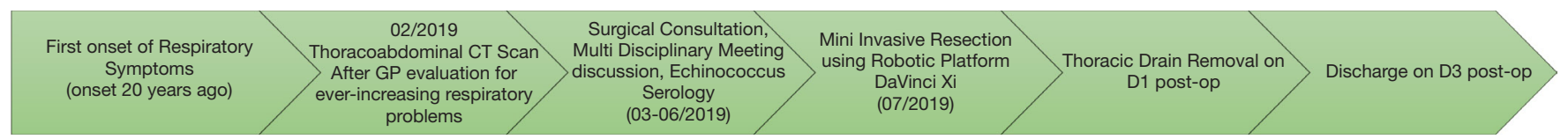

Figure 1 Timeline.

His main symptoms were an ever-increasing difficulty in breathing with nocturnal episodes of acute dyspnea. The patient has never before been investigated for his symptoms. On physical examination there are diminished respiratory sounds on the left base. No palpable abdominal masses or lymphadenopathy is present and the patient is free of any other physical findings.

Workup started with a thoraco-abdominal CT scan which identified a cystic mass $5 \times 6 \mathrm{~cm}$ large, originating from the diaphragm, in contact with the left lobe of the liver, protruding into the inferior mediastinum. No adenopathy was detected. Subsequently a PET CT was performed which did not show any uptake from the suspicious mass. Blood tests were unremarkable apart the fact that echinococcus serology came back positive, establishing the diagnosis of a hydatid cyst and excluding a neoplastic process.

A resection of the cyst was planned with concomitant repair of the diaphragmatic defect due to the chronic paralysis which in the end was probably caused by the cystic infiltration of the diaphragmatic innervation. To our knowledge this is the first surgical video describing a step by step resection of this rare localization of an echinococcal cyst with the synchronous repair of the diaphragm.

The video starts by describing the history, workup and diagnosis, followed by the robotic ports placement. Inspection of the abdominal cavity makes evident the anatomic relations of the cystic mass, which are superimposed in a digital fashion throughout many key steps of the video. Next, we describe a step by step guide to the different parts of the operation. We begin the mobilization of the cystic mass by first liberating the inferior part by its omental adhesions, making sure no noble structures are involved. Next step is the dissection from the diaphragm. We use a monopolar hook diathermy in order to achieve precision. Traction and counter traction is the key for a safe and successful dissection, greatly helped by the robotic arms. As the separation of the mass from the diaphragm continues, an eventual perforation occurs through the diaphragm into the left hemithorax. Sudden loss of pneumoperitoneum occurs, but the patient tolerates well the pneumothorax and we can soon resume the intervention without problems. Dissection continues until the mass is completely liberated from its diaphragmatic attachments. It is placed in an endoscopic bag in the inferior abdomen. The diaphragmatic defect is then closed with two running non-absorbable sutures of Ethibond 0. Next step is the diaphragmatic plication. We perform this plication by placing transverse non-absorbable running sutures of Ethibond 0. Care is taken to remain superficial in order not to grab any pulmonary parenchyma within the sutures. The mass is extracted at the end of the intervention by a small prolongation of the infra-umbilical incision. Finally, the video concludes with the presentation of the anatomopathological results which confirms the diagnosis.

The patient has had an uneventful recovery and was discharged 3 days later. On follow-up he has completely recuperated his respiratory function and reported complete resolution of the acute nocturnal episodes of dyspnea (Figure 1).

\section{Discussion}

This rare presentation of echinococcal disease demonstrates the advantages of robotic platforms. The same operation, would be extremely difficult, if not impossible to be performed with standard laparoscopy. The forces and angulations required during the intervention, would in our opinion resulted in a laparotomy conversion with all the complications and disadvantages this carries. Robotic surgery provides a significant advantage over classical laparoscopy when dealing with intra-abdominal suturing due to its extended degrees of motion, especially when that occurs in difficult angles such as in our case. Robotic diaphragmatic hernia repair has been described in the literature (3-5) but our video is the first in our knowledge, that describes such a repair with a concomitant resection of a hydatid cyst using a robotic platform. Being a case report has on its own its limitations. A standardization on the technique based on our report is of course not possible. Instead we would like to provide an insight on an alternative resection technique when faced with rare localization of these diaphragmatic lesions.

\section{Acknowledgments}

Funding: None. 


\section{Footnote}

Conflicts of Interest: All authors have completed the ICMJE uniform disclosure form (available at https://jovs. amegroups.com/article/view/10.21037/jovs.2020.03.01/ coif). The authors have no conflicts of interest to declare.

Ethical Statement: The authors are accountable for all aspects of the work in ensuring that questions related to the accuracy or integrity of any part of the work are appropriately investigated and resolved. All procedures performed in this study were in accordance with the ethical standards of the institutional and/or national research committee(s) and with the Helsinki Declaration (as revised in 2013). Oral informed consent has been acquired as the patient was not physically available during the creation of this paper.

Open Access Statement: This is an Open Access article distributed in accordance with the Creative Commons Attribution-NonCommercial-NoDerivs 4.0 International License (CC BY-NC-ND 4.0), which permits the noncommercial replication and distribution of the article with the strict proviso that no changes or edits are made and the original work is properly cited (including links to both the formal publication through the relevant DOI and the license). See: https://creativecommons.org/licenses/by-nc-nd/4.0/.

\section{References}

1. Pakala T, Molina M, Wu GY. Hepatic echinococcal cysts: a review. J Clin Transl Hepatol 2016;4:39-46.

2. Gottstein B. Hydatid disease, major tropical syndromes by body system. Systemic infections. Cambridge: Cambridge University Press, 2000:169, section 6.

3. Wei B, Pittman BC Jr. Robotic Morgagni hernia repair: an emerging approach to a congenital defect. J Robot Surg 2019;13:309-13.

4. Arevalo G, Harris K, Sadiq A, et al. Repair of Morgagni hernia in adults with primary closure and mesh placement: first robotic experience. J Laparoendosc Adv Surg Tech A 2017;27:529-32.

5. Jambhekar A, Robinson S, Housman B, et al. Robotic repair of a right-sided Bochdalek hernia: a case report and literature review. J Robot Surg 2018;12:351-5. doi: 10.21037/jovs.2020.03.01

Cite this article as: Popeskou SG, Douissard J, Christou N, Puppa G, Ris F, Buchs NC, Jung MK, Toso C. Robotic resection of a mediastinal hydatic cyst and diaphragmatic repair: a case report. J Vis Surg 2020;6:47. 